\title{
Factores personales, laborales y contextuales del profesional de enfermería que inciden en la presencia de un evento adverso ${ }^{1}$
}

\author{
Zeidy Vargas Bermúdez ${ }^{2}$
}

Institución: Hospital Rafael A. Calderón Guardia

\section{COMO CITAR}

Vargas, Z. (2016). Factores personales, laborales y contextuales del profesional de enfermería que inciden en la presencia de un evento adverso. Rev. Enfermería Actual en Costa Rica, 31, 1-19. http://dx.doi.org/10.15517/revenf.v0i31.24518

\section{RESUMEN}

Introducción. Un evento adverso se define como una situación inesperada que lesiona al paciente y es producto de la interacción con el equipo de salud y la institución. El objetivo del este estudio es describir la opinión de profesionales de enfermería sobre los factores personales, laborales y contextuales que inciden en la presencia de un evento adverso, en el área de medicinas del Hospital Rafael Ángel Calderón Guardia.

Método. La población participante estuvo formada por 38 profesionales que laboran en el área de medicinas. Son criterios de exclusión de eventos adversos las infecciones y úlceras por presión. Las variables estudiadas son características sociodemográficas de la población, conocimiento del concepto de evento adverso y su reporte y la opinión de la condición de infraestructura, equipo, ubicación de usuarios y su distribución como factores relacionados con la aparición de eventos adversos. El estudio es de tipo descriptivo transversal, con un cuestionario validado y autoadministrado.

Resultado. La mayoría de la población tiene conocimiento de la conceptualización de un evento adverso; opina que no hay relación entre la condición de la infraestructura y equipo con la aparición del evento. Manifiesta que la ubicación de pacientes y cantidad asignada por profesional influye en la aparición de eventos adversos.

Conclusión. Los profesionales de enfermería conocen la definición conceptual de evento adverso y consideran que hay relación entre el número de pacientes por profesional, la calidad y ubicación de los mismos en la institución con la presencia de eventos adversos.

Palabras Clave. Eventos-adversos, enfermería, factores-personales, factores-laborales, factores-contextuales

\footnotetext{
${ }^{1}$ Fecha de recepción: 4 de abril del 2016

Fecha de aceptación: 15 de junio del 2016

${ }^{2}$ Enfermera. Subdirectora-Docente. Hospital Rafael Angel Calderón Guardia. Correo electrónico:

zeidyvargas@gmail.com
} 


\title{
Personal, work and contextual factors that influence nurse in the presence of an adverse event ${ }^{1}$
}

Zeidy Vargas Bermúdez ${ }^{2}$

Institution: Rafael A. Calderón Guardia Hospital

\section{CITED}

Vargas, Z. (2016). Personal, work and contextual factors that influence nurse in the presence of an adverse event. Rev. Enfermería Actual en Costa Rica, 31, 1-19. http://dx.doi.org/10.15517/revenf.v0i31.24518

\begin{abstract}
Introduction. An adverse event is defined as an unexpected situation that damages the patient and is a product of the interaction with the health team and the institution. The aim of this study is to describe the opinion of nurses about the personal, labor and contextual factors that influence the presence of an adverse event in the area of medicines Rafael Angel Calderon Guardia Hospital.

Method. The participant population consisted of 38 professionals working in the field of medicine. Exclusion criteria are adverse events infections and pressure ulcers. The variables studied are socio-demographic characteristics of the population, knowledge of the concept of adverse event report itself and the opinion of the condition of infrastructure, equipment, location of users and the distribution of them as a factor related to the occurrence of adverse events. The study is descriptive traversal with a validated self-administered questionnaire. Result. Most of the population is aware of the conceptualization of an adverse event, believe that there is no relationship between the condition of the infrastructure and equipment with the appearance of events. They say that the location of patients and number assigned by professional influences the occurrence of adverse events.

Conclusion. The conceptual definition of adverse event is known not show a reckless behavior before the report. The ratio of patients per professional, quality and location thereof are directly related to adverse events in this institution.
\end{abstract}

Descriptors. Contextual-factors, events-adverse, labor-factors, nursing, personal-factors.

\footnotetext{
${ }^{1}$ Date of receipt: April 4, 2106

Date of acceptance: June 15, 2016

${ }^{2}$ Nursing. Assistant director-Teacher. Rafael Angel Calderon Guardia Hospital. Email. zeidyvargas@ gmail.com
} 


\title{
Pessoal, trabalho e fatores contextuais que influenciam enfermeiros na presença de um evento adverso ${ }^{1}$
}

\author{
Zeidy Vargas Bermúdez ${ }^{2}$
}

\begin{abstract}
Instituição: Hospital Rafael A. Calderón Guardia
CITAÇÃO
\end{abstract}

Vargas, Z. (2016). Pessoal, trabalho e fatores contextuais que influenciam enfermeiros na presença de um evento adverso. Rev. Enfermería Actual en Costa Rica, 31, 1-19. http://dx.doi.org/10.15517/revenf.v0i31.24518

\section{RESUMO}

Introdução. Um evento adverso é definido como uma situação inesperada que danifica o paciente e é um produto da interação com a equipe de saúde e da instituição. O objetivo deste estudo é descrever a opinião dos enfermeiros sobre o trabalho e contextos pessoais, além dos fatores que influenciam a presença de um evento adverso na área de medicamentos do Hospital Rafael Angel Calderón Guardia.

Método. A população participante consistiu de 38 profissionais que trabalham no campo da medicina. Os critérios de exclusão são as infecções e úlceras de decúbito e eventos adversos. As variáveis estudadas são características sócio-demográficas da população, o conhecimento do conceito de relatório de eventos adversos em si e o parecer da condição de infra-estrutura, equipamentos, localização dos utilizadores e a distribuição deles como um fator relacionado à ocorrência de eventos adversos. O estudo é transversal descritivo, com um questionário autoadministrado validado.

Resultado. A maior parte da população tem conhecimento da conceitualização de um evento adverso, acreditam que não existe qualquer relação entre a condição da infra-estrutura e o material com a ocorrência do evento. Eles dizem que a localização dos pacientes e o número atribuído por influências profissionais da ocorrência de acontecimentos adversos.

Conclusão. Os enfermeiros sabem a definição conceitual de evento adverso. Eles não vêem relação entre o número de pacientes por profissional, qualidade e localização dos mesmos na instituição com a presença de eventos adversos.

Palavras-chave. Enfermagem, eventos-adversos, fatores-contextuais, fatores-ocupacionais, fatores-pessoal.

\footnotetext{
${ }^{1}$ Data de recepção: 4 de abril de 2016

Data de aceitação: 15 de junho de 2016

${ }^{2}$ Enfermeira. Assistente do diretor-Mestre. Rafael Angel Calderon Guardia. Correio eletrônico: zeidyvargas@gmail.com
} 


\section{Revista Electrónica Enfermeria Actual en costa Rica}

\section{INTRODUCCIÓN}

Según la opinión de algunos autores como Aranaz y Agra (2010), la cultura de la seguridad del paciente se puede fraccionar a través del tiempo en cuatro momentos históricos. El primero se sitúa en la época de 1692 a.C. con el Código de Hammurabi, Papiro egipcio, considerado el primer conjunto de leyes de la historia que contemplaba algunas específicas para la medicina, con el fin de fomentar el bienestar entre las personas.

El segundo momento se sitúa en la época hipocrática, 450 a.C, con la seguridad en el binomio del médico y la colaboración del paciente. En cuanto al tercero, en los años 1509 -1590, se detallan varios aportes, por ejemplo el de Ambroise Paré, el padre de la cirugía que reconocía y publicaba sus errores, así como Pierre Charles Alexandre, quien -en 1825- refería que era importante documentar lo relacionado con signos y síntomas, respuesta y tratamientos utilizados en los pacientes. Los autores mencionados también mencionan los aportes de Florence Nightingale (1820-1910) quien detectó el riesgo de la atención sanitaria y planteó la necesidad de enseñar a los enfermos y sus familiares a ayudarse a sí para mantener su independencia; de igual modo, incluyen a Ernest Codman (1869-1940) considerado el padre de la calidad asistencial ya que realizaba esfuerzos sistemáticos de seguir a cada uno de los pacientes después de los tratamientos y registrar el resultado final de sus cuidados como médico.

El cuarto momento se sitúa después de los años cincuenta del siglo XX, momento en el que se publicó los primeros estudios sobre los riesgos de la hospitalización y los errores médicos, entre los que se destaca el aporte de anestesistas en los que alertan la necesidad de evaluar los métodos empleados para disminuir las muertes relacionadas con la anestesia. Al respecto, Aranaz y Agra (2010) mencionan que, en esta etapa, los estudios epidemiológicos han realizado aportes valiosos a la metodología para abordar los eventos adversos (EA) y sus determinantes, al tiempo que agregan que en el futuro el reto dependerá del avance tecnológico y la complejidad de la atención, lo cual supone una situación de mayor riesgo para los pacientes. Se incluye ahora el acceso a la información que tiene los ciudadanos, evidenciando que muchas veces los profesionales no están preparados para esos nuevos retos, sin olvidar que en ocasiones no se fortalece la comunicación efectiva entre los diferentes profesionales, causa principal de los EA centinela. Dichos autores manifiestan que la comunicación inefectiva con los usuarios favorece que ocurran los eventos y una mayor frecuencia en discrepancias, demandas y problemas legales.

Los estudios de Baneresa, et al. (2014) demostraron que los EA en hospitales de Catalunya son frecuentes, aunque son bastante prevenibles y generan un impacto considerable en la morbi-mortalidad. El estudio de cohorte retrospectivo de Aranaz (2006) para determinar el impacto y la evitabilidad de EA asociados al cuidado de la salud en hospitales españoles determinó que el $45 \%$ de los pacientes hospitalizados sufrieron un EA, el $39 \%$ lo sufrió moderado y el $16 \%$ severo, con una incidencia de muerte del 4,4\%. El 84,2\% pudo prevenirse, debido a su relación con el diagnóstico: "infecciones nosocomiales" $(56,6 \%)$ y "cuidado" (56\%). El estudio demuestra que los EA no solo impactan al usuario que los sufre, sino que también aumentan el costo hospitalario debido al consumo de medicamentos y estancias hospitalarias prolongadas, lo que justifica esfuerzos para su prevención y mejoramiento de la atención en salud. 


\section{Revista Electrónica Enfermeria Actual en costa Rica}

Por su parte, Bañeres, Cavero, López, Orrego y Suñol (s.f.) menciona que hasta un $16 \%$ de los pacientes sufre algún daño durante la hospitalización, que en un $70 \%$ provoca un daño temporal y en un $14 \%$, la muerte del paciente. En los Estados Unidos, López y Gomez (2011) aportan que los eventos adversos son la octava causa de mortalidad en dicho país, mientras que en Colombia hay una prevalencia de 13,1 \% en 12 hospitales que participaron en el estudio sobre estas complicaciones.

De acuerdo con Gómez, et al. (2011) la relevancia en torno al tema inició a partir de la publicación del libro "Errar es humano" del Instituto de Medicina de la Academia Nacional de Ciencias de los Estados Unidos, en el que la OMS argumenta que "77\% de los EA se relacionan con medicamentos adulterados y de baja calidad, el mal estado de la infraestructura y de los equipos médicos, el escaso control de las infecciones y la falta de cultura hacia la seguridad del paciente" (p.98).

Sobre el costo económico, Allue, et al. (2014) evidencia que un 6,8\% del gasto total en hospitales españoles se dirige al tratamiento de EA los cual podría reducirse mediante la mejora de la calidad y la seguridad del sistema de salud. Así mismo Vlayen, et al. (2012), en una revisión sistemática sobre la incidencia y evitabilidad de EA que requirió que el paciente fuese hospitalizado en unidades de cuidado intensivo, muestra que a pesar que no se pudo identificar una incidencia clara debido a la heterogeneidad en los estudios, se identificó que en un 1,1\% a 37,2\% los usuarios requirieron asociado a EA admisión en unidades de cuidado intensivo.

Por su parte, Hernández, Mendoza, Cabrera y Ortega (2006), Tomás y Gimena (2010), Queiroz, Queiroz y Tanferri-de-Brito-Paranagua (2012) mencionan que en Gran Bretaña, el costo de EA evitables es de aproximadamente US\$ 1500 millones por año, solamente por la ocupación adicional de cama hospitalaria. Así también, la evidencia científica respalda que la seguridad del paciente y prevención de EA dependen de la interacción y el equilibrio permanente de múltiples actuaciones del sistema sanitario y de sus profesionales.

En general los estudios relacionados con eventos adversos advierte que las intervenciones realizadas al paciente en el ámbito hospitalario implican un riesgo por la complejidad de la atención y los procedimientos cada vez más invasivos. En este caso, relaciona los EA con la infraestructura, calidad de medicamentos, deficiencia en eliminación de desechos, control de infecciones, deficiente actuación del personal por falta de conocimientos, motivación o insuficiencia de recursos. En el ámbito hospitalario, los EA no son exclusivos para una disciplina; no obstante, asociados a la naturaleza del quehacer de cada una, se pueden individualizar respecto de la atención de enfermería, como en el caso de la flebitis, úlceras por presión y caídas que, según Parra, Camargo-Figuera y Rey (2012), son los de mayor incidencia registrados. Como dato relevante refiere que se identificó un 16,5\% como incidencia de eventos adversos, de los que un $63 \%$ de la población presentó uno; $25 \%$, dos y un 9\%, tres, siendo las úlceras por presión el más frecuente.

Por otra parte, Hernández, et al. (2006) realizó un estudio descriptivo, retrospectivo y lineal en el que analizó eventos registrados: los resultados aportaron que el 31,1\% corresponde a errores en la administración de medicamentos como causa más frecuente, el 38,3\% se atribuye a la alteración en el desarrollo de procedimientos de enfermería en general; un 6,94\%, a error en la administración de hemoderivados y el 3,85\%, a quejas por maltrato. 


\section{Revista Electrónica Enfermeria Actual en costa Rica}

En la disciplina de enfermería se han realizado análisis relacionados con la percepción, cultura y algunos tipos de sucesos adversos, pero es necesario el desarrollo de más estudios al respecto, sobre todo en Costa Rica, donde no se identificó alguno relacionado con esta temática a pesar de estar incluidos con el tema de "seguridad" en la institución, incluido en la Sección de Enfermería en los Estándares de Calidad en la atención del paciente (Conejo, Monge, Romero, Solano y Arias, 2006), documento que presenta los estándares mínimos básicos que se deben ofrecer en la Atención de Enfermería, fundamentados en las políticas, objetivos estratégicos y metas de la institución. En este caso, respecto del paciente hospitalizado, se destaca las infecciones nosocomiales, continuidad del cuidado, seguridad del paciente. En la misma línea se cuenta también con el Protocolo para la Prevención y el tratamiento de las úlceras (Loaiza, et al., 2015) liderado por la Coordinación Nacional de Enfermería en Costa Rica.

Por otra parte, la literatura aporta ejemplos de sistemas implementados en algunos países para garantizar la vigilancia de EA y el cuidado. Al respecto, Hernández, et al. (2006) define un cuidado seguro como "aquel que además de cumplir con las necesidades y expectativas del paciente, al ser desarrollado alcanza un estado de logro que beneficia en todos los sentidos a la persona que es atendida." (p.72)

A nivel de Latinoamérica y América Central se identificó un trabajo publicado por los autores López y Gómez (2011) en el que participó un total de 58 hospitales de diferentes países (Costa Rica, México, Perú, Colombia y Argentina). En el estudio, denominado IBEAS, se determinó la prevalencia de suceso adverso en algunos hospitales de dichas naciones.

Propiamente en el Hospital Rafael Ángel Calderón Guardia, el Departamento de Enfermería tiene establecido desde hace varios años el reporte de EA que consiste en completar un instrumento que en el que se registra los datos de identificación del paciente, área y causa de la hospitalización, fecha, hora, descripción del evento, datos del testigo, diagnóstico provisional, tratamiento de emergencia, disposición final relacionada con el evento y nombre del médico tratante y del profesional que realiza el reporte. El documento es completado por los profesionales de enfermería y ha estado enfocado en su mayoría al reporte voluntario de eventos asociados a caídas o lesiones físicas del paciente, ya que es mínimo el reporte de otro tipo de eventos, lo cual obliga a fortalecerlo tomando en cuenta las recomendaciones basadas en la experiencia en otros países, así como la implementación en una forma interdisciplinaria en la institución.

En cuanto al presente estudio, es pionero en el tema en lo que respecta a la exploración a través de la opinión de los profesionales de enfermería en un hospital nacional clase A, de cuyos resultados la investigadora considera plantear un proyecto de sensibilización respecto del tema, mejorar el proceso de abordaje de los y las pacientes y el registro de EA, así como aplicar un sistema que identifique los riesgos y desarrollar estrategias que minimicen la incidencia de los EA mediante la prevención. La hipótesis descriptiva del trabajo es los factores personales laborales y contextuales del enfermero (a) durante la atención influyen en la presencia de sucesos adversos en los pacientes hospitalizados en el área de medicinas.

Por último, su objetivo general fue describir los factores personales, laborales y contextuales del profesional de enfermería que influyen en la presencia de eventos adversos en los pacientes hospitalizados durante la atención en el área de medicinas del Hospital Rafael Calderón Guardia. 


\section{Revista Electrónica Enfermeria Actual en costa Rica}

\section{www.revenf.ucr.ac.cr}

\section{MATERIALES Y MÉTODOS}

En lo concerniente a la población, participaron todos los profesionales de enfermería que laboran en el Área de Medicinas, por lo cual no se delimita criterios de exclusión o inclusión de participantes.

Esta investigación se perfila desde el enfoque descriptivo y transversal al establecer variables de estudio en términos de medidas cuantitativas de valores absolutos y descripción de las variables sin elementos de comparación, durante el mes de octubre del año 2015. Para efectos de este estudio, no se considerará las úlceras por presión e infecciones nosocomiales, definidas por algunos autores como sucesos adversos, debido a que cuentan con un sistema de notificación y abordaje establecido a través del Comité de Prevención de infecciones asociadas al cuidado de la salud y un Comité de Prevención y Tratamiento de las úlceras por presión que existe en el hospital, sin dejar de lado que pueden confundir los criterios de la población en estudio para efectos de la investigación.

Las variables planteadas se relacionan con elementos sociodemográficos, conocimiento del concepto de EA, identificación de los tipos y la conducta y abordaje manifestado por los profesionales ante la presencia del mismo y la opinión que manifiesta sobre la relación entre el estado de la infraestructura, equipo, aspectos organizacionales, la distribución y ubicación de pacientes como causal o factor relacionado con el EA. El instrumento de recolección de datos se creó a partir de las variables de estudio y fue validado con un $10 \%$ del equivalente a la población en estudio en otra área del hospital. La tabulación de la información la ejecutó una colaboradora de la investigadora principal, mediante el sistema SPSS.

\section{Consideraciones éticas}

La población fue invitada a participar a través de un consentimiento informado, anónimo, en el que autorizaban su colaboración, así como el uso de los datos proporcionados para fines estadísticos y de análisis descritos en la investigación. De acuerdo con los resultados, se propondrá un plan de mejoras que favorezca la calidad de atención de los pacientes hospitalizados en este aspecto en el Departamento de Enfermería. La investigación contó con el Aval N CLOBI 37-10-2015 del Comité de Bioética de la institución donde se realizó el estudio. El desarrollo del trabajo no representa ningún conflicto de intereses para la investigadora.

\section{RESULTADOS}

Los datos están cuantificados con la participación total de 38 profesionales de enfermería del área de medicina, debido a que cuatro se encontraban en periodo de vacaciones al momento del estudio. Los resultados se presentan tomando en cuenta los valores absolutos.

En cuanto a la variable sociodemográfica, la mayoría tiene edades entre los 27 y 32 años con un total de 27 personas; en cuanto al sexo, 22corresponde al femenino y 16, al masculino. Se registra como mayoría el lugar de residencia San José (21), seguido de Cartago (10) y un restante de otras provincias (7). Respecto del grado académico, 30 cuentan con licenciatura y 8, con maestría. En lo concerniente al puesto en el que se desempeñan, 32 laboran como enfermeros 1 y 6, como enfermeros 2. Por último, en relación con los años laborados, 6 tienen 


\section{Revista Electrónica Enfermeria Actual en costa Rica}

menos de un año; 5, de 1 a menos de tres años; 21, entre 5 y menos de 10 años y 6 cuentan con más de 10 años de laborar en la institución.

En la tabla 1 se observa que en cuanto al concepto de EA, 32 personas lo identifican como accidentes que ocurren durante la atención de salud; 25, como lesiones involuntarias y 20, como lesiones físicas o psicológicas que ocurren al paciente durante la estancia hospitalario, lo cual evidencia que conocen el concepto; sin embargo, 18 profesionales no los identifican como tal.

En cuanto al concepto de EA como lesiones que pueden conducir a la muerte o incapacidad, solamente 16 personas lo definen de tal forma.

Tabla 1

HCG: distribución de la opinión de los profesionales de enfermería del área de medicina respecto del concepto de evento adverso, 2016 (Frecuencias absolutas)

\begin{tabular}{l|c|c|c|}
\hline \multicolumn{1}{c|}{ Opinión de los profesionales de enfermería } & \multicolumn{3}{c|}{ Frecuencia } \\
\cline { 2 - 4 } & Sí & No & Total \\
\hline Son lesiones involuntarias & 25 & 13 & 38 \\
\hline Complicaciones de la enfermedad subyacente & 12 & 26 & 38 \\
\hline Accidentes que ocurren durante la atención en salud & 32 & 6 & 38 \\
\hline $\begin{array}{l}\text { Lesiones que pueden conducir a la muerte o } \\
\text { incapacidad }\end{array}$ & 16 & 22 & 38 \\
\hline $\begin{array}{l}\text { Lesiones físicas o psicológicas que ocurren al } \\
\text { paciente durante la estancia hospitalaria }\end{array}$ & 20 & 18 & 38 \\
\hline
\end{tabular}

Fuente: Elaboración propia, datos del instrumento de opinión, 2015

En la tabla 2, se observa que la mayoría de la población identifica claramente las caídas, lesiones y/o accidentes, extravasaciones e infiltración y flebitis como EA. Cabe destacar que la omisión en la administración de tratamientos y el retiro involuntario de dispositivos no son incluidos en el concepto. 


\section{Revista Electrónica Enfermeria Actual en costa Rica}

\section{Tabla 2}

HCG: distribución de la opinión de los profesionales de enfermería del área de medicina respecto de la correspondencia de un evento adverso, 2015

(Frecuencias absolutas)

\begin{tabular}{l|c|c|c}
\hline \multirow{2}{*}{\multicolumn{1}{c|}{ Tipo de evento adverso }} & \multicolumn{3}{c}{ Frecuencias } \\
\cline { 2 - 4 } & Sí & No & Total \\
\hline Úlceras por presión & 26 & 12 & 38 \\
\hline Infecciones & 26 & 12 & 38 \\
\hline Caídas & 37 & 1 & 38 \\
\hline Lesiones y o accidentes & 31 & 7 & 38 \\
\hline Errores en medicamentos & 20 & 18 & 38 \\
\hline $\begin{array}{l}\text { Omisión en administración de } \\
\text { tratamiento }\end{array}$ & 11 & 27 & 38 \\
\hline Retiro involuntario de dispositivos & 9 & 29 & 38 \\
\hline Extravasaciones vasculares & 30 & 8 & 38 \\
\hline $\begin{array}{l}\text { Infiltración medicamentos } \\
\text { intravenosos }\end{array}$ & 30 & 8 & 38 \\
\hline Flebitis & 30 & 8 & 38 \\
\hline Ninguno & 0 & 0 & 38 \\
\hline
\end{tabular}

Fuente: Elaboración propia, datos del instrumento de opinión, 2015

$\mathrm{Al}$ solicitar acerca de la conducta para reportar el EA, 38 indican que lo reporta sin temor a represalias, 5 lo hacen con temor, mientras que 1 prefiere no hacerlo.

Los datos aportados por la población muestran que en su mayoría realizan una serie de actividades en respuesta al evento, entre las que se destaca el reporte al médico (35), jefatura de enfermería (35), vigilancia de signos de complicación (35), el establecimiento de un plan de atención de enfermería (31), registro el evento en la nota de enfermería (35), reporte escrito establecido en el departamento (34), seguimiento al usuario (33), comunicación al personal del siguiente turno (36), y seguimiento de las indicaciones médicas (1).

En cuanto a la opinión de los criterios organizacionales establecidos ante la presencia de un EA en la unidad de trabajo, mencionan la ejecución de las siguientes actividades: reporte a la jefatura inmediata (36) reporte al médico tratante (35), vigilancia de los signos de complicaciones (32), registro en la nota de enfermería (34), reporte escrito (36), seguimiento al paciente y el evento (31), seguimiento de indicaciones médicas (37) y otras actividad sin especificar (30). El único aspecto que tiene menor porcentaje de ejecución corresponde a la elaboración del plan de atención de enfermería, acerca del que 28 menciona que lo diseña posterior a que ocurra el EA, mientras que 7 no lo realizan; sin embargo, se observa un número significativo que sí lo elabora. 


\section{Revista Electrónica Enfermeria Actual en costa Rica}

Respecto de la opinión de los participantes en el estudio de la condición de la infraestructura, 1 la considera excelente; 5 , buena; 24 , regular y 7 , en mala condición. Además, 22 opinan que la condición de los equipos es mala, aunque 13 participantes afirman que los equipos están en buenas condiciones, mientras que 30 , los clasifica como en excelente estado.

En cuanto al vínculo entre el estado de la infraestructura con el EA, la mayoría (21) lo anota como poco frecuente, 15 piensa que es muy frecuente y 2 , que es infrecuente. En lo referente a condición del equipo y el EA, 28 opinan que es poco frecuente; 8 , muy frecuente y 1 , infrecuente.

Acerca de cuán frecuente influye el área física con la aparición de EA, 25opinan que es poco frecuente y 13, que es muy frecuente. En relación con los equipos, 21 considera que es poco frecuente; 15, muy frecuente y 2 , infrecuente.

Luego, 26 participantes refieren que la ubicación de pacientes según su condición de salud influye en la prevención de EA, 10 opinan que algunas veces influye y 2, que no influye. Finalmente, 33 sujetos expresaron que la cantidad de pacientes asignados por profesional ha influido en la presencia de EA, mientras que 4 opinan que algunas veces y 1 , que no influye.

\section{DISCUSIÓN}

La seguridad del paciente se constituye en una política establecida en las instituciones de salud, la cual incluye la detección y prevención de EA como estrategias de gestión, directamente relacionadas con la calidad de atención brindada por los diferentes profesionales y personal de apoyo. Los EA han fundamentado una manera objetiva de identificar el porcentaje de calidad establecidos en dichas instituciones.

Por la naturaleza del trabajo, los profesionales de enfermería se exponen a riesgos relacionados con EA derivados de su quehacer, lo que invita a identificar la conducta que muestran ante el riesgo o presencia de un suceso adverso en el paciente, ya que reportarlo dependerá de esa percepción, sin olvidar el seguimiento que se brinde al usuario y, más importante aún, el fortalecimiento del proceso para la prevención a partir de experiencias previas y el conocimiento de diferentes aspectos relacionados con el tema.

El tema de seguridad es básico en el desempeño de la labor de enfermería y está directamente ligado a la profesión, puesto que los profesionales en el área permanecen mucho tiempo con los pacientes, lo que hace más pronta la detección de errores relacionados con su quehacer o el de otro profesional del equipo de salud. En relación con lo anterior, y debido a que el reporte de los EA depende de la percepción, la cultura y la noción de seguridad, así como del concepto de acciones punitivas al respecto, Gaitán-Duarte, Gómez-Sánchez, y Eslava-

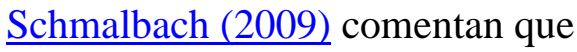

La evaluación de los determinantes de los eventos adversos requiere de la aproximación cuantitativa para describir la magnitud y fuerza de asociación de los factores y de la aproximación cualitativa que permite tomar en cuenta aspectos culturales, tanto de los diferentes contextos sociales como organizacionales, que no son extrapolables a diferentes lugares 


\section{Revista Electrónica Enfermeria Actual en costa Rica}

específicamente el hecho de no tomar en cuenta las consideraciones socioculturales y los factores técnicos que inciden en un bajo reporte de eventos adversos. La reacción inicial del personal de salud caracterizada por la resistencia, el temor, negación de las complicaciones y la espera de acciones punitivas, ha sido previamente descrita en diferentes culturas (p.750).

Otros autores mencionan la importancia de identificar y analizar los EA para mejorar la calidad de atención y los procesos de gestión en las instituciones, tal como Hernández, et al. (2006), quien señala que

En la Séptima Reunión Anual del Foro Europeo de las Asociaciones de Enfermería y la Organización Mundial de la Salud, se enfatizaron puntos importantes en relación con la seguridad de los pacientes, en estos puntos se reconoció que la seguridad del paciente conlleva un análisis y comprensión de los EA y una estimación real del problema. Esto implica generar una cultura donde el personal de enfermería pueda informar sobre problemas y errores con el fin de que en sus centros interinstitucionales puedan adoptar políticas y procedimientos con el propósito de reducir la incidencia de riesgos y sus consecuencias. Esta cultura involucra un cambio de paradigma, el cual consiste en adoptar una actitud más activa dentro del área hospitalaria, colaborando con el resto de las disciplinas sanitarias en el desarrollo de iniciativas dirigidas a la prevención de errores (p.71).

Lo descrito justifica el porqué de realizar estudios con los profesionales de enfermería respecto de definir conceptualmente el EA, la cultura que se tiene ante el reporte y abordaje de este una vez sucedido el hecho y la descripción de si identifica algunos aspectos relacionados, causales o que influyen en la aparición de tales complicaciones. En este aspecto la población participante de esta investigación manifestó que reporta las caídas y lesiones asociadas con libertad, pero no señaló la omisión de medicamentos o retiro involuntario de dispositivos como EA, lo cual será necesario investigar mediante otro estudio. Dichas conductas son mencionadas por Gómez, et al. (2011) quien, basado en la literatura, corrobora la necesidad de implementar estrategias de educación en tema de seguridad y reporte oportuno de EA, ya que diversos estudios apuntan al bajo reporte de los casos relacionados por el temor a acciones disciplinarias al respecto.

Al realizar un análisis de aspectos que influyen directamente en la aparición de EA resulta interesante valorar la percepción u opinión de los profesionales de enfermería, sobre todo en las instituciones de salud nacionales y partiendo de que algunos podrían atribuirse a factores personales, laborales o contextuales en el medio hospitalario. Al respecto, Urbina (2011) apunta que

como resultado de esta relación se potencializa la responsabilidad profesional implícita en el acto del cuidado. Lo que demanda una formación de enfermeros con una visión científica de la profesión, preocupados por el bien de la humanidad, capaces de brindar cuidados oportunos, de calidad y libres de riesgo (p.241)

Lo mencionado indica que las intervenciones para el cuidado de la salud de las personas, implica riesgos a partir de la unión compleja de procesos, tecnologías e interacciones humanas, ya que a pesar de contribuir con acciones favorables, también incluyen posibilidades de práctica insegura para la ocurrencia de EA. Es así como en la actualidad, y a pesar del avance de la tecnología y el conocimiento científico, aun es aplicable la frase de Florence Nightingale, citada por Miyares (2014) quien mencionó: "parece sorprendente, que lo primero que haya que pedirle a un hospital es que no cause ningún daño" 


\section{Revista Electrónica Enfermeria Actual en costa Rica}

Para autores como Queiroz, et al.(2012), la esencia del quehacer del profesional de enfermería competente es la responsabilidad de la coordinación y administración de todo el proceso de atención al paciente, su asistencia debe ser realizada eficientemente, garantizando la calidad del cuidado mediante la aplicación del conocimiento científico actualizado y, principalmente, respetando los valores, deseos y considerando la satisfacción, del paciente y sus familiares, lo cual incluye lo relacionado con los EA, sus causas, control y factores de riesgo para implementar medidas preventivas y tratamientos eficaces que reduzcan y eviten los fallos identificados, de modo que se minimice su impacto y contribuya al desarrollo de una práctica segura, un aspecto relevante para el sistema de salud, el cual no está excepto de tales riesgos.

En la literatura se observa la variedad de factores que pueden influir en la aparición de EA que, para $\underline{\text { Aranaz, }}$ et al.(2006) y Zarate-Grajalesa, et al.(2015) más allá de los relacionados con factores personales del equipo de salud, se refieren a fallos del sistema (organización y gestión) dentro del que se señala elementos relacionados con condiciones laborales, como sobrecarga de trabajo, indefinición de funciones, debilidades en la formación técnica y profesional, así como con el control de las tareas, la estandarización de protocolos y procedimientos. En el área de comunicación, menciona el uso de tecnología de punta y en los planes de prevención y corrección de instalaciones, de igual modo, señala faltas relacionadas con la actividad o actos inseguros como omisión, distracción, errores, fallos de atención, incumplimiento de procedimiento, no adhesión a protocolos, falta de habilidades del personal, presión por el tiempo y la falta de atención, distracción, preocupación, sobrecarga, aburrimiento de los profesionales de salud, así como la fatiga y el estrés. Al respecto, según los datos analizados en este estudio se observa que los profesionales refieren que la cantidad de pacientes asignados así como su ubicación están relacionadas con la aparición de eventos adversos, lo que concuerda con aspectos mencionaos por los autores.

Sin embargo también hay que considerar lo que anotan Ferreira, Fort y Chiminelli (2015) y Bernal y Garzón $\underline{(2008)}$ quienes, además de los aspectos señalados, mencionan la aparición e incidencia de errores asociados al estado de salud de los pacientes y las características de gestión administrativa de ciertas áreas, entre lo que se destaca la ausencia o insuficientes recursos materiales y equipo, así como poca asertividad en las relaciones interpersonales de los integrantes del equipo laboral. Un ejemplo de lo anterior se da en lugares como las unidades de cuidados intensivos, las cuales representan un factor predisponente para la ejecución y realización de eventos adversos.

Las cargas de trabajo han sido aspectos relacionados con la aparición de EA analizados por algunos autores como Zago, Vasconcelos, Quitério y Daud-Gallotti (2014); Barrios, Catoni, Valenzuela y Fernández (2012), quienes afirman que una mayor proporción de enfermeras/paciente disminuye las tasas de EA y la mortalidad, un aspecto que debe ser considerado al realizar los análisis pertinentes, máxime dentro de la gestión del recurso humano, dado que los EA fueron atribuidos al exceso de trabajo, situación que incide en el número de días de hospitalización y el riesgo de muerte de los pacientes.

La posición de tales autores coincide -hasta cierto punto- con las emitidas por los profesionales participantes en esta investigación, quienes en general consideran que la gestión administrativa, así como la distribución y características de los pacientes son factores que pueden incidir en la presencia o ausencia de eventos adversos, sin dejar de lado las características epidemiológicas de los usuarios que atienden y el cuidado de enfermería. 


\section{Revista Electrónica Enfermeria Actual en costa Rica}

$\mathrm{Al}$ conocer los aspectos descritos, los cuales coinciden con los elementos considerados en este estudio, se puede trabajar en estrategias conjuntas y multidisciplinarias para minimizar el impacto causal de EA y, por ende, mejorar la calidad de atención y la seguridad de los usuarios del sistema hospitalario nacional.

Otro factor importante en el tema de EA y la seguridad de los pacientes es lo referente a la implementación de la vigilancia y procesos para abordar y prevenir dichas complicaciones: entre los pasos para investigar y analizar un incidente clínico, en la bibliografía revisada se identifica que tanto en un error como un EA a nivel institucional se debe platear un protocolo tanto para la identificación y seguimiento del evento como la implementación de medidas preventivas basado en etapas en las que, según Taylor-Adams \&Vincent (2012) y Bernal y Garzón (2008) que conlleva lo siguiente: identificación y decisión de investigar para lo que debe haber una cultura institucional de hablar libremente de las fallas, sin miedo al castigo, donde no se sancione el error pero si el ocultamiento, b. selección del equipo investigador, c. adquisición y organización de la información, d. orden del incidente en forma cronológica, e. Identificación de las tareas o actividades de riesgo. i. Detección de los elementos indirectos asociados al incidente g. Presentación de las sugerencias y plan de respuesta d. Elaboración de las recomendaciones y plan de acción, a lo que se debe agregar los Comités de Calidad enfocados en esos aspectos (Bernal y Garzón, 2008).

De igual modo, en la literatura también se documenta organizaciones (SENSAR, 2015) para realizar un abordaje sistemático de los EA con el objetivo de que guíen el accionar, ya que el propósito último de la respuesta a un EA debe ser mejorar los procesos asistenciales para evitar que se repita.

Otro ejemplo de sistemas de notificación y seguimiento de EA es el planteado por Parra, et al. (2012), y el de Buriticá (2015) cuya herramienta, Tigger Tool, permite la detección activa de EA; también está el descrito por Grigorov, et al. (2008), el cual consiste en un sistema de gestión clínica del riesgo en una unidad hospitalaria. El objetivo de dicho proyecto fue crear un sistema de notificación y manejo del error, intentando hacerlo evidente y palpable, sin ánimo punitivo, anónimo y dirigido a evitar y corregir la repetición sistemática de errores, mientras se aprovecha los avances tecnológicos a través de herramientas de eficacia demostrada, la integración de otras estrategias acordes y la presencia del personal de enfermería las 24 horas del día como fuente primaria y oportuna en el reporte de los EA.

Modelos como los descritos, se constituyen en herramientas valiosas en materia de seguridad en la atención del paciente al contemplar estrategias relacionadas con la identificación de las acciones inseguras relacionadas con acciones u omisiones que pueden causar un incidente o evento adverso, además de que permite identificar factores predisponentes a realizar actividades de riesgo asociadas con cargas de trabajo y cansancio, desconocimiento, experiencia, control o orientación escasa o ambigua, cultura organizacional tensa, cambios acelerados en la institución, sistemas de comunicación deficientes, además de programas ineficientes en la estructura y equipos, cuyo impacto en la aparición de EA es directo. Lo anterior también se puede observar en las opiniones emitidas por los participantes cuando aluden a la cantidad de pacientes que tienen a su cargo como factor relacionado con la aparición de EA, a lo que se agrega que, aun cuando no relacionan la condición de los equipos e infraestructura con los EA, son considerados en regular y mala condición.

Siguiendo con las posiciones de los autores mencionados, Hernández, et al. (2006) manifiesta que el conocer y reportar los EA es clave para su corrección y para la seguridad en la atención del paciente lo que 


\section{Revista Electrónica Enfermeria Actual en costa Rica}

permite hablar en términos reales de garantía de calidad en el cuidado de enfermería. Por su parte, Mena (2008) refiere que analizar los errores como una fuente de aprendizaje y cambio permite mantener una mejoría continua en la calidad de la atención, aspecto fundamental que debe ser considerado en las políticas tanto locales como institucionales a nivel de la CCSS, ya que como lo afirman Aibar y Aranaz (2003)

los objetivos del establecimiento de un sistema de notificación de errores y sucesos adversos son: aprender de la experiencia ajena, valorar la evolución de los progresos en prevención, detectar riesgos emergentes relacionados con la aparición de nuevas técnicas diagnósticas y terapéuticas, y en definitiva, contribuir a mejorar la seguridad del paciente. (p.199)

Es importante resaltar que para mejorar la cultura de la calidad desde la perspectiva de reducción de EA, un sistema de identificación no debe producir miedo por castigo o represalia que resulte de este. Al respecto Aibar y Aranaz (2003) mencionan que

Las características más sobresalientes en este tipo de sistemas son: confidencialidad de manera que la identificación del paciente, el informador y la institución no debe ser nunca revelada. Independencia de la autoridad con capacidad de castigar al informador o su organización. Los datos deben ser analizados y los informes valorados por expertos que consideren las circunstancias del suceso reportado de una forma ágil para hacer llegar las recomendaciones a las personas en forma precisa, especialmente si se han identificado riesgos importantes. Las recomendaciones deben centrarse en cambios en los procesos y los recursos, y el comportamiento de los individuos (p. 200).

En relación con lo anterior, López y Gomez (2011) plantean unas recomendaciones e instrumentos de evaluación para implementar herramientas en programas de atención segura en instituciones de salud, basados en un estudio realizado en varios países que incluyó Costa Rica. Los autores destacan algunas prácticas de implementación las cuales, en primera instancia, deben contar con el apoyo de la gerencia tanto a nivel general como local; luego, es imprescindible desarrollarlas por etapas, lo cual incluye un plan de capacitación, el desarrollo de la política de seguridad, estructurar un formato de reporte de EA y/ o incidentes, plan de mejoras, implementar una base de datos electrónica que permita hacer un seguimiento y sacar indicadores. De acuerdo con lo descrito, es necesario que la institución donde se llevó a cabo el estudio, en específico en el Departamento de Enfermería, se fortalezca un análisis del proceso establecido para el reporte de los EA, con el fin de incorporar los elementos que aseguren no solo el reporte de todo tipo de evento, sino que asegure un reporte sin temor a represalias y -ante todo- que sirva de insumo para el desarrollo de estrategias que aseguren la disminución de incidencia de errores y mejore la seguridad de los usuarios. La confidencialidad, proceso de reporte, seguimiento y análisis oportuno de los datos se constituye en la prioridad del trabajo de acuerdo con lo expuesto en la literatura al respecto.

\section{CONCLUSIÓN}

La opinión de los profesionales de enfermería de un hospital clase A de la seguridad social, relacionado a los factores personales, laborares y contextuales que inciden en la aparición de un evento, revela que a pesar que 


\section{Revista Electrónica Enfermeria Actual en costa Rica}

se conoce la definición conceptual de EA y se manifiesta una conducta no temeraria ante el reporte, se enfocan básicamente en situaciones como son las caídas. Un porcentaje bajo establece una relación con la condición de infraestructura y equipos con el evento adverso, situación contraria a la relación de pacientes por profesional y la calidad y ubicación de los mismos, de lo que se colige que estos últimos aspectos sí están directamente relacionados con los eventos adversos en esta institución.

\section{AGRADECIMIENTO}

A las autoridades del Hospital R. A. Calderón Guardia, a los profesionales de enfermería que participaron en la investigación y a la colega Dra. Ligia Patricia Rojas Valenciano Coordinadora Programa de Colaboración para la Investigación de Enfermería Basada en la Evidencia de Costa Rica CIEBE-CR, por su asesoría para el desarrollo del estudio.

\section{CONFLICTO DE INTERESES}

La autora declara no tener conflicto de intereses para el desarrollo de esta investigación.

\section{REFERENCIAS BIBLIOGRÁFICAS}

Aibar, C. y Aranaz, J. (2003). ¿Pueden evitarse los sucesos adversos relacionados con la atención hospitalaria? An. sis. sanit. Navar. , 26 (2), 195-209.

Allue, N, Chiarelloa, P. , Delgadoe, E.B., Castellsb, X., Giraldo, P., Martíneze, N., Sarsanedasg, E. y Cots, F. (2014). Impacto económico de los eventos adversos en los hospitales españoles a partir del Conjunto Mínimo Básico de Datos. Gac Sanit , 28 (1), 48,54.

Aranaz, J. y Agra, Y. (2010). La cultura de seguridad del paciente:del pasado al futuro en cuatro tiempos. Medicina Clínica, 135.

Aranaz, J. (2006). Impacto y evitabilidad de eventos adversos en los hospitales publicos españoles: resultados del Estudio Nacional Español de acontecimientos Adversos (ENEAS). Recuperado de: http://www.msssi.gob.es/organizacion/sns/planCalidadSNS/pdf/excelencia/opsc_sp2.pdf

Aranaz, J., Aibar, C., Galan, A., Limon, R., Requena, J., Alavarez, E.E., y Gea, M.T. (2006). La asistencia sanitaria como factor de riesgo: los efectos adversos ligados a la práctica de enfermería. Gac Sanit , 20, 4147.

Baneresa, J., Orregoa, C., Navarrob, L., Casas, L., Banque, M., y Sunol, R. (2014). Epidemiología de los eventos adversos hospitalarios en Catalunya: un primer paso para la mejora de la seguridad del paciente. Medicina Clínica, 3-10. 


\section{Revista Electrónica Enfermeria Actual en costa Rica}

Bañeres, J., Cavero, E., López, L., Orrego, C. y Suñol, R. (s.f). Sistemas de registro y notificación de incidentes y eventos adversos. Recuperado de:

http://www.msssi.gob.es/organizacion/sns/planCalidadSNS/pdf/excelencia/opsc_sp3.pdf

Barrios, S., Catoni, M.I., Valenzuela, V. y Fernández, F. (2012). ¿Existe relación entre dotación de personal de enfermería, eventos adversos y mortalidad? Enferm Nefrol, 13 (3), 163-169.

Bernal, D.C. y Garzón, N. (2008). Eventos adversos durante la atencion de enfermeria en unidades de cuidado intensivo. Recuperado de http://www.javeriana.edu.co/biblos/tesis/enfermeria/tesis44.pdf

Buritica, C.A. (2015). Eventos adversos: uno de los más importantes dolores del sistema de salud. Revista Universitas Científica, 12-15.

Conejo, A., Monge, J., Romero, J., Solano, N., y Arias, A. M. (2006). Manual normativo del programa institucional de estándares de salud dirigido a los tres niveles de atención. Recuperado de: http://www.ccss.sa.cr/arc/publicaciones/23/Manual_programa_institucional_estandares_de_salud.zip

Ferreira, A., Fort, Z. y Chiminelli, V. (2015). Eventos adversos en salud y cuidados de enfermería: la seguridad del paciente desde la experiencia del profesional. Enferm, Florianópolis, , 24 (2), 310-5.

Gaitán-Duarte, H., Gómez-Sánchez, P.I. y Eslava-Schmalbach, J. (2009). Actitudes del personal en la vigilancia de eventos adversos intrahospitalarios en Colombia. Rev. salud pública, 11 (5), 745.746.

Gómez, O., Arenas, W., González, L., Garzón, J., Mateus, E. y Soto, A. (2011). Cultura de seguridad del paciente por personal de enfermería en Bogotá, Colombia. Ciencia y Enfermería XVII , 3, 97.111.

Grigorov, I., Fernández, S., Ferrera, A., Baena, M., Valentí, P., Tomas, M., Poua, J., Aguilara, J.L. y Gargoulas, F. (2008). Sistema de gestión de incidentes críticos y eventos adversos en los ámbitos área, servicio o unidad hospitalarios. Puesta en marcha en tres fases. Rev Calidad Asistencial. , 23 (5), 230-5.

Hernández, C., Mendoza, R., Cabrera, P. y Ortega, V. (2006). Eventos adversos en el cuidado de enfermería. Revista Mexicana de Enfermería Cardiológica, 14 (2), 70-74.

Loaiza, C., Monge, J., Solís, M., Álvarez, M., Córdoba, H. A., Moreno, A., Sequeira, V., Garita, Y. y Artiaga, S. (2015). Manual Técnico de Prevención y Tratamiento de Úlceras por Presión. Recuperado de http://www.ccss.sa.cr/publicaciones?pagina=8

Lopez, J. y Gómez, J. (2011). Recomendaciones para la implementacion de herramientas en programas de atencion segura en instituciones prestadoras de salud (IPS). Arch Med (Manizales), 11 (2), 169-182.

Mena, P. (2008). Error médico y eventos adversos. Revista Chilena de Pediatría, 79 (3), 319-326. 


\section{Revista Electrónica Enfermeria Actual en costa Rica}

Mendoza, E., Cabrera, F., Ortega, C. y Hernández, A. (2006). Eventos adversos en el cuidado de enfermería. Revista Mexicana de Enfermería Cardiológica, 14 (2), 70-74.

Miyares, A. (2014). Caídas en pacientes hospitalizados: un evento adverso evitable. Recuperado de: http://repositorio.unican.es/xmlui/bitstream/handle/10902/5228/MiyaresOlavarriaA.pdf?sequence=1

Parra, D.I., Camargo-Figuera, F.A. y Rey, R. (2012). Eventos adversos derivados del cuidado de enfermería: flebitis, úlceras por presión y caídas. Enferm. glob., 11 (28).

Queiroz, A.L., Queiroz, E. dos S., Weber, J., y Tanferri-de-Brito-Paranaguá, T. (2012). Reacciones adversas: indicadores de resultados según la percepción de las enfermeras de un hospital centinela. Enfermería Global (27), 186-197.

SENSAR. (2015). Recomendaciones para la respuesta institucional de un evento adverso. Revista de Calidad asistencial , 31 (1), 42-54.

Taylor-Adams, S. y Vincen, C.(s.f.). Protocolo de Londres Incidentes clinicos.Recuperado de https://www.minsalud.gov.co/sites/rid/Lists/BibliotecaDigital/RIDE/DE/CA/PROTOCOLO_DE_LONDRE S_INCIDENTES\%20CLINICOS.pdf

Tena, C., Arroyo,G., Victoria, R.,Manuell, G., Sánchez, J., Hernández, L., Campos, E., Muñoz, J., Ortega, C., Medina, S., Ibarra, G. y Hernández, G. (2006). Recomendaciones especificas para enferemria relacionadas con la prevencion de caidas de pacientes durante la hospitalizacion. Revista CONAMED, Vol. 11, Núm. 5, enero - marzo, 2006,11 (5).

Tomás, S. y Gimena, I. (2010). La seguridad del paciente en urgencias y emergencias. An. Sist. Sanit. Navar, 33 (1), 131-148.

Urbina, O. (2011). Competencias de Enfermería para la seguridad del paciente. Revista Cubana de Enfermería, 27 (3), 239-247.

Vlayen, A., Verelst, S., Bekkering, G.E, Schrooten, W., Hellings, J. \& Claes, N. (2012). Incidence and preventability of adverse events requiring intensive care admission: a systematic review. Journal of Evaluation in Clinical Practice, 18 (2), 485.97.

Zago, M.C., Vasconcelos, E., Quitério, L.M. y Daud-Gallotti, R. (2014). Sobrecarga de trabalho da Enfermagem e incidentes e eventos adversos em pacientes internados em UTI. Rev Bras Enferm. , 67 (5), 692-9.

Zárate-Grajalesa, R., Olvera-Arreolab, S., Hernández-Cantoralc, A., Hernández, S. Sánchez-Angelese, S., Valdez, R., Pérez-Lópezg, M.T. y Zapién-Vázquezh, M.A. (2015). Factores relacionados con eventos 


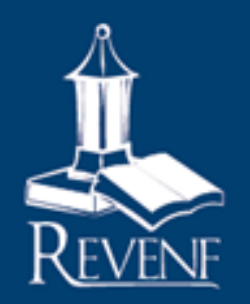

\section{Revista Electrónica Enfermeria Actual en costa Rica}

adversos reportados por enfermeria en unidades de cuidados intensivos. Proyecto multicéntrico. Enfermeria Universitaria, 12 (2), 63-72. 